\title{
Maximal Translational Equivalence Classes of Musical Patterns in Point-Set Representations
}

\author{
Tom Collins ${ }^{1}$ and David Meredith ${ }^{2}$ \\ ${ }^{1}$ Department of Computational Perception, \\ Johannes Kepler University Linz, Austria \\ ${ }^{2}$ Department of Architecture, Design and Media \\ Technology, Aalborg University, Denmark \\ ${ }^{1}$ tom.collins@jku.at, ${ }^{2}$ dave@create.aau.dk
}

\begin{abstract}
Representing musical notes as points in pitch-time space causes repeated motives and themes to appear as translationally related patterns that often correspond to maximal translatable patterns (MTPs) [1]. However, an MTP is also often the union of a salient pattern with one or two temporally isolated notes. This has been called the problem of isolated membership [2]. Examining the MTPs in musical works suggests that salient patterns may correspond more often to the intersections of MTPs than to the MTPs themselves. This paper makes a theoretical contribution, by exploring properties of patterns that are maximal with respect to their translational equivalence classes (MTEC). We prove that a pattern is MTEC if and only if it can be expressed as the intersection of MTPs. We also prove a relationship between MTECs and so-called conjugate patterns.
\end{abstract}

Keywords: pattern discovery, motivic analysis, repetition in music, pointset patterns, music analysis, geometric music representations

\section{Introduction}

In this paper, we address an aspect of the problem of computing a structural description of a passage of music when given a multi-dimensional point-set representation of the passage as input (as used, for example, in [1-11]). We are particularly concerned with computing structural descriptions that identify all (and only) the occurrences of perceptually salient or analytically interesting patterns that are stated more than once, possibly at different transpositions. Such patterns typically correspond to motives, themes, and sections.

We assume throughout this paper that each note in the passage to be analysed is represented as a two-dimensional point in pitch-time space (see Figures 1 and 2 for examples). However, the results presented here actually apply to point sets of any finite dimension, and for any domain where it is useful to represent data as multi-dimensional point sets.

When a pattern is repeated, it is commonly transposed modally by a certain number of scale steps, rather than chromatically by a certain number of semitones. We therefore generally assume in this paper that, if the input passage 
is tonal, then the pitch of each note is represented by an integer indicating its morphetic or "diatonic" pitch $[1,8]$, which corresponds to the note's position on the staff, in contrast to its chromatic pitch, which is usually represented by its MIDI note number. Given a MIDI file of a passage of tonal music as input, the morphetic pitches of the notes in the passage can typically be computed with a high degree of accuracy using a pitch-spelling algorithm such as PS13s1 [8, 10]. Using morphetic instead of chromatic pitch allows for many types of modal transposition to be found using fast, robust, exact-matching algorithms, rather than slower, less precise, approximate-matching algorithms (see [1, pp. 329-330]).

Meredith et al. [1] propose that perceptually salient and/or analytically interesting repeated patterns in music often correspond to what they call maximal translatable patterns (or MTPs, see section 2). Given a point-set representation of a passage of music in pitch-time space, as described above, the MTP for a particular vector is the set of points (i.e., notes) that can be translated by that vector to give other points in the point-set representation (see Figure 2 for some examples). It can be shown that the sum of the cardinalities of all the transpositionally distinct MTPs for non-zero vectors for a dataset containing $n$ points is less than or equal to $n(n-1) / 2$, implying that the number of such MTPs is substantially less than the $2^{n}$ distinct subsets of $D$. Thus, if the set of perceptually salient repeated patterns in a musical passage is generally a subset of the MTPs for a passage, then the space of candidate patterns to be searched by an algorithm aiming to find only the interesting patterns can be greatly reduced.

A

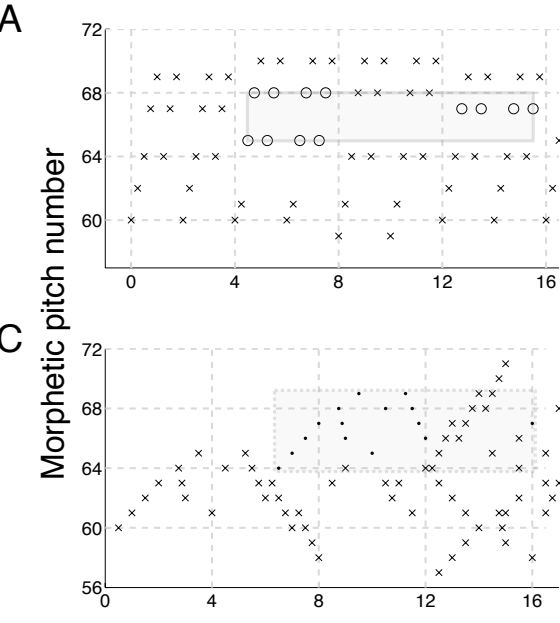

$\mathrm{B}$

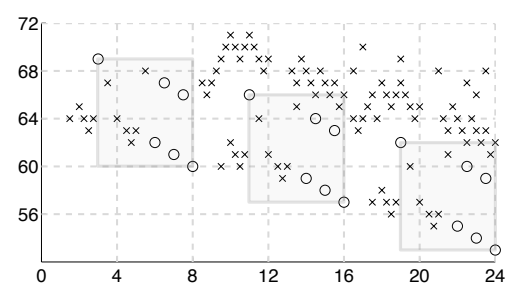

D

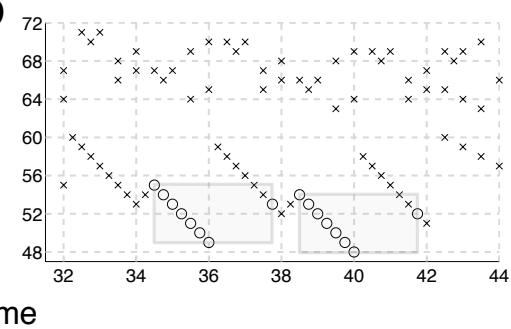

Fig. 1. MTPs from works in the first book of J.S. Bach's Das Wohltemperierte Clavier that exhibit the problem of isolated membership. (A) Prelude in C major (BWV 846); (B) Fugue in C sharp major (BWV 848); (C) Fugue in C major (BWV 846); (D) Fugue in $\mathrm{C}$ minor (BWV 847). 
Meredith et al. [1] also propose that those patterns that are perceived as most salient, generally tend to be compact, in the sense that their bounding boxes do not usually contain many non-pattern points. This hypothesis was recently confirmed experimentally by Collins et al. [5]. However, Collins et al. [2] observe that it is not uncommon for an MTP to be the union of a salient, compact pattern with a small number of temporally isolated notes that prevent the MTP as a whole from corresponding to a perceptually salient musical pattern. Collins et al. [2] call this the problem of isolated membership. Figure 1 shows some examples of MTPs in works from the first book of Johann Sebastian Bach's (1685-1750) Das Wohltemperierte Clavier that suffer from this problem.

One way of solving this problem would be to filter out the less compact patterns in the output of an algorithm such as SIATEC that finds MTPs, as described in [9]. An alternative strategy, implemented in Collins et al.'s SIACT algorithm [2], is to "trawl" each MTP and return subsets of lexicographically consecutive pattern points (assuming the time dimension is given priority) that have compactness and cardinality greater than specifiable thresholds.

Another way of removing isolated points from an MTP is to calculate its intersection with a second MTP that contains fewer or different isolated points. Here, we explore properties of the class of patterns that are intersections of MTPs, showing that this is also the class of patterns that are maximal with respect to their translational equivalence classes or MTEC. A relationship is also proved between MTEC patterns and another interesting class of patterns called conjugate patterns [4]. We do not demonstrate definitively that MTECs solve the problem of isolated membership, but focus instead on developing some new theory. To begin, we review some definitions and lemmas relating to maximal translatable patterns.

\section{A Review of Maximal Translatable Patterns}

The material in this section consists mainly of rearrangements of definitions and lemmas from [1], with new examples. They are necessary for building new theory in later sections.

Definition 1. A point set $D$ is a non-empty, finite subset of $\mathbb{R}^{k}$. A pattern $P$ is a subset of $D$, and, unless stated otherwise, non-empty and not equal to $D$.

Definition 2. The difference set for a point set $D$, written $\Delta(D)$, is defined by

$$
\Delta(D)=\{\boldsymbol{e}-\boldsymbol{d} \mid \boldsymbol{d}, \boldsymbol{e} \in D\} .
$$

Working with $\Delta(D)$ is somewhat inefficient, since $\boldsymbol{u} \in \Delta(D)$ implies $-\boldsymbol{u} \in$ $\Delta(D)$. For $\boldsymbol{d}=\left(d_{1}, d_{2}, \ldots, d_{k}\right), \boldsymbol{e}=\left(e_{1}, e_{2}, \ldots, e_{k}\right) \in D$, we say that $\boldsymbol{d}$ is less than $\boldsymbol{e}$, denoted by $\boldsymbol{d}<\boldsymbol{e}$, if and only if there exists an integer $1 \leq j \leq k$ such that $d_{j}<e_{j}$, and $d_{i}=e_{i}$ for $1 \leq i<j$. Then the reduced difference set for a point set $D$, written $\Delta^{*}(D)$, is defined by

$$
\Delta^{*}(D)=\{\boldsymbol{e}-\boldsymbol{d} \mid \boldsymbol{d}, \boldsymbol{e} \in D, \text { and } \boldsymbol{d}<\boldsymbol{e}\} .
$$


Definition 3. For an arbitrary vector $\boldsymbol{v} \in \mathbb{R}^{k}$ and an arbitrary point set $D \subset$ $\mathbb{R}^{k}$, the maximal translatable pattern (MTP) of the vector $\boldsymbol{v}$ in the point set $D$ is

$$
\operatorname{MTP}(\boldsymbol{v}, D)=\{\boldsymbol{d} \in D \mid \boldsymbol{d}+\boldsymbol{v} \in D\} .
$$

Example 1. An excerpt of a piece by Anton Bruckner (1824-1896) is shown in Figure $2 \mathrm{~A}$, with the line bounding a pattern that contains 9 notes. Switching to the point-set representation $D$, shown in Figure 2B, the MTP of the vector $\boldsymbol{v}=(4,-2)$ in $D$ is shown as black dots. Two solid arrows indicate the vector $\boldsymbol{v}$, and that members of $\operatorname{MTP}(\boldsymbol{v}, D)$ are translatable in $D$ by $\boldsymbol{v}$.

Does $P=\operatorname{MTP}(\boldsymbol{v}, D)$ correspond to the pattern of interest from Figure 2A? Not exactly: there is an extra, temporally isolated point with coordinates $(3.5,62)$, which also happens to be translatable in $D$ by $\boldsymbol{v}=(4,-2)$. This is an example of the problem of isolated membership [2]. An algorithm that returns all MTPs in a point set $D$ has been defined [1], but MTPs are affected by isolated membership. Addressing this problem would make the algorithmic discovery of repeated patterns more effective, removing the need to check output MTPs for isolated members. A second MTP, for the vector $\boldsymbol{u}=(2,-1)$, is shown as empty circles. We return to it in Example 5, but again it contains extra points.
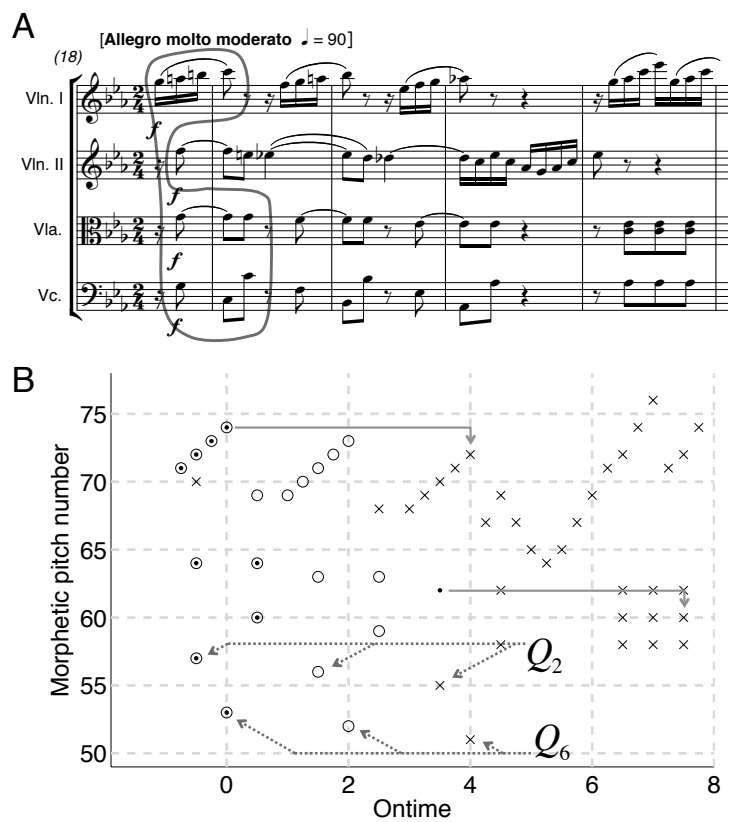

Fig. 2. (A) Bars 18-21 (with upbeat) of the Rondo in C minor for String Quartet (WAB 111) by Anton Bruckner. A nine-note pattern, repeated twice at lower pitch heights, is bounded by the line; (B) Point-set representation for the excerpt from Figure 2A. Two MTPs are shown as empty circles and black dots respectively. 
Lemma 1. Let $\boldsymbol{v} \in \mathbb{R}^{k}$ be an arbitrary vector, and $D \subset \mathbb{R}^{k}$ an arbitrary point set. The maximal translatable pattern of $\boldsymbol{v}$ in $D$, denoted $\operatorname{MTP}(\boldsymbol{v}, D)$, is nonempty if and only if $\boldsymbol{v} \in \Delta(D)$, where $\Delta(D)$ is the difference set for $D$.

Proof. See [1].

Definition 4. For an arbitrary vector $\boldsymbol{v} \in \mathbb{R}^{k}$ and an arbitrary pattern $P$, the translation of the pattern $P$ by the vector $\boldsymbol{v}$ is defined by

$$
\tau(P, \boldsymbol{v})=\{\boldsymbol{p}+\boldsymbol{v} \mid \boldsymbol{p} \in P\} .
$$

Let $P, Q$ be arbitrary patterns. We say that $P$ is translationally equivalent to $Q$, written $P \equiv_{\tau} Q$, if and only if there exists some vector $\boldsymbol{v} \in \mathbb{R}^{k}$ such that $Q=\tau(P, \boldsymbol{v})$. It can be shown that $\equiv_{\tau}$ is an equivalence relation in the proper mathematical sense (reflexivity, symmetry, and transitivity).

For an arbitrary point set $D \subset \mathbb{R}^{k}$ and an arbitrary, non-empty pattern $P \subseteq D$, the translational equivalence class of $P$ in $D$ is defined by

$$
\operatorname{TEC}(P, D)=\left\{Q \subseteq D \mid Q \equiv_{\tau} P\right\} .
$$

Example 2. Letting $P$ be the set of nine points in Figure 2B that are black dots surrounded by circles, the translational equivalence class of $P$ in $D$ consists of three sets in total. This is perhaps easiest to see by focusing on the part of $P$ in Figure 2B that forms a diagonal line. There is $P$ itself, a second occurrence appearing as empty circles to the right, and a third occurrence appearing as crosses further to the right.

Definition 5. Working with $\operatorname{TEC}(P, D)$ is somewhat inefficient, since if $|P|=l$ and $|\operatorname{TEC}(P, D)|=m$, then $l m$ vectors need to be stored in memory. For a point set $D \subset \mathbb{R}^{k}$ and a pattern $P \subseteq D$, the translators of $P$ in $D$ are given by

$$
T(P, D)=\left\{\boldsymbol{v} \in \mathbb{R}^{k} \mid \tau(P, \boldsymbol{v}) \subseteq D\right\} .
$$

Now only $l+m$ vectors need be stored in memory to represent the same information, or only $l+m-1$ if we remove the ever-present zero vector from $T(P, D)$.

Example 3. With $P$ and $D$ defined as in Example 2, the translators of $P$ in $D$ are $\mathbf{0}=(0,0), \boldsymbol{u}=(2,-1)$, and $\boldsymbol{v}=(4,-2)$.

\section{Maximal Translational Equivalence Classes}

Material in this and subsequent sections is novel. The purpose of this section is to introduce another type of maximal repeating pattern, and to show how it relates to maximal translatable patterns.

Definition 6. Let $P$ be a non-empty pattern in a point set $D$, and let the translational equivalence class of $P$ in $D$, denoted $\operatorname{TEC}(P, D)$, have $m$ elements. We 
say that $P$ is maximal with respect to its translational equivalence class (or $M T E C$ for short) if for each non-empty subset $S$ of $D \backslash P$,

$$
|\operatorname{TEC}(P \cup S, D)|<m .
$$

That is, however the pattern $P$ is extended to include arbitrary new elements from the point set $D$, the resulting translational equivalence class has fewer members than $\operatorname{TEC}(P, D)$.

Remark 1. The proofs below assume that $S$ contains one arbitrary element, usually denoted by $\boldsymbol{e}$. This is a valid assumption to make, for if it is possible to extend the pattern $P$ by two, three etc. elements in a way that does not reduce the cardinality of the resulting translational equivalence class, then there exist extensions consisting of one element.

Example 4. Figure 3A contains a point set $D=\{\boldsymbol{a}, \boldsymbol{b}, \boldsymbol{c}, \boldsymbol{d}, \boldsymbol{e}, \boldsymbol{f}, \boldsymbol{g}\}$. The pattern $P=\{\boldsymbol{a}, \boldsymbol{b}\}$, which is shown as empty circles and occurs 4 times, is MTEC. We can verify $P$ is MTEC by extending it, and observing that the number of occurrences for each extension $P^{\prime}$ is less than 4 (see first two columns of Table 1). In Figure 2B, the pattern consisting of dots surrounded by circles is also MTEC, but this would take much longer to verify, suggesting that we need to gain a better understanding of how to discover MTEC patterns.

A

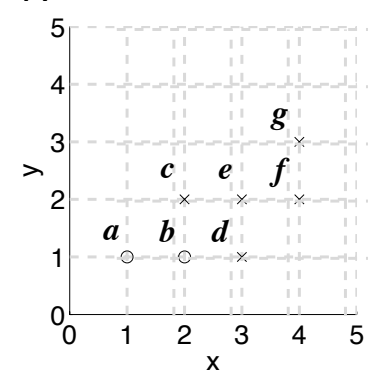

B

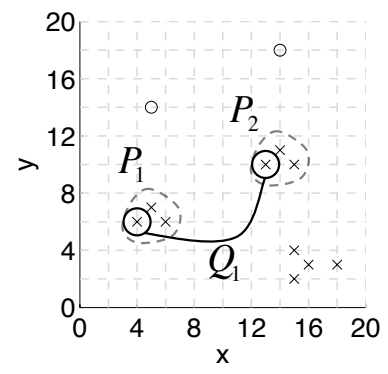

Fig. 3. (A) An example of a pattern $P=\{\boldsymbol{a}, \boldsymbol{b}\}$ that is MTEC, but is not an MTP; (B) An example of conjugate patterns. Pattern $P_{1}$, containing 3 points, is indicated by the dashed bounding line. Its conjugate pattern $Q_{1}$, containing 2 points, is indicated by the solid bounding lines.

Lemma 2. Let $P$ be a non-empty pattern in a point set $D$, with $\operatorname{TEC}(P, D)$ having $m$ elements. If $P$ is maximal with respect to $\operatorname{TEC}(P, D)$ (i.e., $P$ is MTEC) then for an arbitrary member $Q \in \operatorname{TEC}(P, D)$ such that $Q \neq P, Q$ is also maximal with respect to $\operatorname{TEC}(P, D)$ (i.e., $Q$ is also $M T E C$ ). 
Table 1. Column 1 shows extensions of pattern $P=\{\boldsymbol{a}, \boldsymbol{b}\}$ from Figure 3A, and column 2 shows $m=\left|\operatorname{TEC}\left(P^{\prime}, D\right)\right|$, the number of occurrences of $P^{\prime}$ in $D$. Columns 3 and 4 show the maximal translatable pattern in $D$ for each non-zero translator $\boldsymbol{v}$ of $P$.

\begin{tabular}{l|l||l|l}
\hline$P^{\prime}$ & $m$ & $\boldsymbol{v}$ & $\operatorname{MTP}(\boldsymbol{v}, D)$ \\
\hline$\{\boldsymbol{a}, \boldsymbol{b}, \boldsymbol{c}\}$ & 3 & $(1,0)$ & $\{\boldsymbol{a}, \boldsymbol{b}, \boldsymbol{c}, \boldsymbol{e}\}$ \\
$\{\boldsymbol{a}, \boldsymbol{b}, \boldsymbol{d}\}$ & 2 & $(1,1)$ & $\{\boldsymbol{a}, \boldsymbol{b}, \boldsymbol{d}, \boldsymbol{e}\}$ \\
$\{\boldsymbol{a}, \boldsymbol{b}, \boldsymbol{e}\}$ & 3 & $(2,1)$ & $\{\boldsymbol{a}, \boldsymbol{b}, \boldsymbol{c}\}$ \\
$\{\boldsymbol{a}, \boldsymbol{b}, \boldsymbol{f}\}$ & 1 & & \\
$\{\boldsymbol{a}, \boldsymbol{b}, \boldsymbol{g}\}$ & 1 & & \\
\hline
\end{tabular}

Proof. Suppose otherwise, that there exists $e \in D \backslash Q$ such that

$$
|\operatorname{TEC}(Q \cup\{\boldsymbol{e}\}, D)| \geq m,
$$

and try to derive a contradiction. We know $Q=\tau(P, \boldsymbol{v})$ for some vector $\boldsymbol{v}$. So

$$
\begin{aligned}
\boldsymbol{e} \in D \backslash Q & \Rightarrow \boldsymbol{e} \in D \backslash \tau(P, \boldsymbol{v}) \\
& \Rightarrow \boldsymbol{e} \notin \tau(P, \boldsymbol{v}) \\
& \Rightarrow \boldsymbol{e} \notin\{\boldsymbol{p}+\boldsymbol{v} \mid \boldsymbol{p} \in P\} \\
& \Rightarrow \boldsymbol{e}-\boldsymbol{v} \notin P .
\end{aligned}
$$

Let $\boldsymbol{d}=\boldsymbol{e}-\boldsymbol{v}$. There are two cases: either $\boldsymbol{d} \in D$; or $\boldsymbol{d} \notin D$.

- If $\boldsymbol{d} \in D$ then $\operatorname{TEC}(Q \cup\{\boldsymbol{e}\}, D)$ and $\operatorname{TEC}(P \cup\{\boldsymbol{d}\}, D)$ are different ways of writing the same set. As $\boldsymbol{d} \in D \backslash P$, we can exploit the maximality of $P$ with respect to $\operatorname{TEC}(P, D)$, giving

$$
|\operatorname{TEC}(Q \cup\{\boldsymbol{e}\}, D)|=|\operatorname{TEC}(P \cup\{\boldsymbol{d}\}, D)|<m .
$$

This is a contradiction to (8).

- If $\boldsymbol{d} \notin D$ then

$$
P \cup\{\boldsymbol{d}\} \notin \operatorname{TEC}(Q \cup\{\boldsymbol{e}\}, D), \quad \text { as } P \cup\{\boldsymbol{d}\} \text { is not a subset of } D .
$$

So $\operatorname{TEC}(Q \cup\{\boldsymbol{e}\}, D)$ has lost one member compared with $\operatorname{TEC}(P, D)$. But has it gained any members?

$$
\begin{aligned}
A \in \operatorname{TEC}(Q \cup\{\boldsymbol{e}\}, D) \Rightarrow & A=\tau(Q \cup\{\boldsymbol{e}\}, \boldsymbol{x}) \quad \text { for some vector } \boldsymbol{x} \\
& =\{\boldsymbol{q}+\boldsymbol{x} \mid \boldsymbol{q} \in Q\} \cup\{\boldsymbol{e}+\boldsymbol{x}\} \\
\Rightarrow & A \backslash\{\boldsymbol{e}+\boldsymbol{x}\}=\tau(Q, \boldsymbol{x}) \\
\Rightarrow & A \backslash\{\boldsymbol{e}+\boldsymbol{x}\} \in \operatorname{TEC}(Q, D) \\
\Rightarrow & A \backslash\{\boldsymbol{e}+\boldsymbol{x}\} \in \operatorname{TEC}(P, D) .
\end{aligned}
$$


This shows the answer is 'no', that for each $A \in \operatorname{TEC}(Q \cup\{\boldsymbol{e}\}, D)$, there is (at least) one member $A \backslash\{\boldsymbol{e}+\boldsymbol{x}\} \in \operatorname{TEC}(P, D)$. So $\operatorname{TEC}(Q \cup\{\boldsymbol{e}\}, D)$ has not gained any members compared with $\operatorname{TEC}(P, D)$.

Taken together with (14), this implies $|\mathrm{TEC}(Q \cup\{\boldsymbol{e}\}, D)|<m$, a contradiction to (8).

In both cases a contradiction to the opening supposition has been derived. We conclude that if $P$ is maximal with respect to $\operatorname{TEC}(P, D)$ then for an arbitrary member $Q \in \operatorname{TEC}(P, D)$ such that $Q \neq P, Q$ is maximal with respect to $\operatorname{TEC}(P, D)$.

Remark 2. The argument in lines (15)-(17) will be needed again, so we call it the no-gains argument. Lemma 2 says that if $P \in \operatorname{TEC}(P, D)$ is maximal with respect to its translational equivalence class, then so is each member of $\operatorname{TEC}(P, D)$. This means that the concept of a maximal translational equivalence class (or "MTEC" used as a noun rather than an adjective) is well defined.

Lemma 3. Let $D$ be a point set with difference set $\Delta(D)$, and $P=\operatorname{MTP}(\boldsymbol{u}, D)$ for some $\boldsymbol{u} \in \Delta(D)$. (By Lemma 1, $P$ is non-empty.) Then $P$ is maximal with respect to its translational equivalence class (MTEC).

Proof. Let $1<|\operatorname{TEC}(P, D)|=m<|D|$, and let

$$
\begin{aligned}
\operatorname{TEC}(P, D) & =\left\{P_{0}, P_{1}, \ldots, P_{m-1}\right\}, \\
P_{0} & =P, \\
P_{i} & =\tau\left(P, \boldsymbol{x}_{i}\right), \quad i=0,1, \ldots, m-1 .
\end{aligned}
$$

Note $\boldsymbol{x}_{0}=\mathbf{0}$. Now $P=\{\boldsymbol{d} \in D \mid \boldsymbol{d}+\boldsymbol{u} \in D\}$, so without loss of generality, assume $\boldsymbol{x}_{1}=\boldsymbol{u}$. Letting $\boldsymbol{e} \in D \backslash P$,

$$
P \cup\{\boldsymbol{e}\}=\{\boldsymbol{d} \in D \mid \boldsymbol{d}+\boldsymbol{u} \in D\} \cup\{\boldsymbol{e}\}
$$

We know $\boldsymbol{e}+\boldsymbol{u} \notin D$, otherwise $P$ would not be a maximal translatable pattern. Therefore $P_{1} \cup\{\boldsymbol{e}+\boldsymbol{u}\} \notin \operatorname{TEC}(P \cup\{\boldsymbol{e}\}, D)$.

So TEC $(P \cup\{\boldsymbol{e}\}, D)$ has lost at least one member compared with $\operatorname{TEC}(P, D)$. But has it gained any members? The answer is 'not compared with $\mathrm{TEC}(P, D)$ ', by the no-gains argument (15)-(17) (with each occurrence of $Q$ replaced by $P$ ).

Therefore, $|\operatorname{TEC}(P \cup\{\boldsymbol{e}\}, D)|<m$, and by definition, $P$ is MTEC.

Remark 3. The converse does not hold in general. That is, $P$ can be maximal with respect to its translational equivalence class $\operatorname{TEC}(P, D)$, but none of the members satisfy $Q=\operatorname{MTP}(\boldsymbol{v}, D)$, where $Q \in \operatorname{TEC}(P, D)$ and $\boldsymbol{v} \in \Delta(D)$ are arbitrary. An example of such a pattern $P=\{\boldsymbol{a}, \boldsymbol{b}\}$ was shown in Figure 3A. We demonstrated in Example 4 that $P$ is MTEC. Columns 3 and 4 of Table 1 show the maximal translatable pattern in $D$ for each non-zero translator of $P=\{\boldsymbol{a}, \boldsymbol{b}\}$. None are equal to $P$, so $P$ is not an MTP. 
Theorem 1. A non-empty pattern is maximal with respect to its translational equivalence class if and only if that pattern is equal to the intersection taken over the maximal translatable patterns of its translators. In symbols,

$$
P \subseteq D \text { is } M T E C \Longleftrightarrow P=\bigcap_{\boldsymbol{v} \in T(P, D)} \operatorname{MTP}(\boldsymbol{v}, D),
$$

where $T(P, D)$ is the set of translators of $P$ in $D$.

Proof. ' $\Rightarrow$ ' First we suppose that $P \subseteq D$ is non-empty and MTEC. Let $T(P, D)=\left\{\boldsymbol{v}_{1}, \boldsymbol{v}_{2}, \ldots, \boldsymbol{v}_{m}\right\}$ and

$$
S=\bigcap_{i=1}^{m} \operatorname{MTP}\left(\boldsymbol{v}_{i}, D\right)
$$

$P$ is translatable by each of the vectors $\boldsymbol{v}_{1}, \boldsymbol{v}_{2}, \ldots, \boldsymbol{v}_{m}$, so belongs to each of $\operatorname{MTP}\left(\boldsymbol{v}_{1}, D\right), \operatorname{MTP}\left(\boldsymbol{v}_{2}, D\right), \ldots, \operatorname{MTP}\left(\boldsymbol{v}_{m}, D\right)$, and hence belongs to their intersection also. Therefore it is a subset of $S$. If it is a proper subset of $S$ (i.e., not equal to $S)$, then there exists $\boldsymbol{e} \in S \backslash P$ such that $\operatorname{TEC}(P \cup\{\boldsymbol{e}\}, D)$ has as many elements as $\operatorname{TEC}(P, D)$, which is a contradiction to $P$ being MTEC. It follows that $S=P$, which is what we wanted to show.

' $\Leftarrow$ ' Now we suppose that $P \subseteq D$ is non-empty and expressible as the intersection taken over the maximal translatable patterns of its translators

$$
P=\bigcap_{i=1}^{m} \operatorname{MTP}\left(\boldsymbol{v}_{i}, D\right)
$$

where $T(P, D)=\left\{\boldsymbol{v}_{1}, \boldsymbol{v}_{2}, \ldots, \boldsymbol{v}_{m}\right\}$.

We wish to show that $P$ is $M T E C$, so let $e \in D \backslash P$ be arbitrary, and try to derive

$$
|\operatorname{TEC}(P \cup\{\boldsymbol{e}\}, D)|<|\operatorname{TEC}(P, D)|=m .
$$

For at least one $j \in\{1,2, \ldots, m\}, \boldsymbol{e} \notin \operatorname{MTP}\left(\boldsymbol{v}_{j}, D\right)$. Otherwise $\boldsymbol{e} \in P$, implying $\boldsymbol{e} \notin D \backslash P$, which is a contradiction. So without loss of generality, assume $\boldsymbol{e} \notin$ $\operatorname{MTP}\left(\boldsymbol{v}_{1}, D\right)$. We know $\boldsymbol{e}+\boldsymbol{v}_{1} \notin D$, otherwise $\boldsymbol{e} \in \operatorname{MTP}\left(\boldsymbol{v}_{1}, D\right)$. Therefore $\tau\left(P \cup\{\boldsymbol{e}\}, \boldsymbol{v}_{1}\right) \notin \operatorname{TEC}(P \cup\{\boldsymbol{e}\}, D)$.

So TEC $(P \cup\{\boldsymbol{e}\}, D)$ has lost at least one member compared with $\operatorname{TEC}(P, D)$. $B u t$ has it gained any members? The answer is 'not compared with TEC $(P, D)$ ', by the no-gains argument (15)-(17) (with each occurrence of $Q$ replaced by $P$ ). This shows that $P$ is MTEC.

Example 5. Two MTPs are shown in Figure 2B: the MTP of $\boldsymbol{u}=(2,-1)$ as empty circles and the MTP of $\boldsymbol{v}=(4,-2)$ as dots. From Theorem 1, we know that the intersection of these MTPs, which consists of the dots surrounded by circles, is MTEC. This intersection also corresponds exactly to the pattern of interest from Figure 2A. So for this excerpt at least, computing an MTEC pattern solves the problem of isolated membership. 


\section{Conjugate Patterns and Their Relation to MTECs}

Suppose there are $m$ translationally equivalent occurrences in some point set of a pattern $P$ whose size is $l$. This section shows that there must be another pattern $Q$ consisting of $m$ points that has at least $l$ occurrences in the same point set.

Definition 7. Let $P$ be a pattern in a point set $D$, with translational equivalence class $\operatorname{TEC}(P, D)=\left\{P_{1}, P_{2}, \ldots, P_{m}\right\}$. For an occurrence $P_{i} \in \operatorname{TEC}(P, D)$, let $P_{i}=\left\{\boldsymbol{p}_{i, 1}, \boldsymbol{p}_{i, 2}, \ldots, \boldsymbol{p}_{i, l}\right\}$. The conjugacy array $\boldsymbol{J}_{P, D}$ for the pattern $P$ in the point set $D$ is defined by

$$
\boldsymbol{J}_{P, D}=\left(\begin{array}{cccc}
\boldsymbol{p}_{1,1} & \boldsymbol{p}_{1,2} & \cdots & \boldsymbol{p}_{1, l} \\
\boldsymbol{p}_{2,1} & \boldsymbol{p}_{2,2} & \cdots & \boldsymbol{p}_{2, l} \\
\vdots & \vdots & \ddots & \vdots \\
\boldsymbol{p}_{m, 1} & \boldsymbol{p}_{m, 2} & \cdots & \boldsymbol{p}_{m, l}
\end{array}\right)
$$

Each row of $\boldsymbol{J}_{P, D}$ constitutes an element of $\operatorname{TEC}(P, D)$, but what about the columns of $\boldsymbol{J}_{P, D}$ ? Letting $Q=\left\{\boldsymbol{p}_{1,1}, \boldsymbol{p}_{2,1}, \ldots, \boldsymbol{p}_{m, 1}\right\}$ be the set of points from the first column, each column of $\boldsymbol{J}_{P, D}$ constitutes an element of $\operatorname{TEC}(Q, D)$. It is said that $P$ and $Q$ are conjugate patterns, and that $\operatorname{TEC}(P, D)$ and $\operatorname{TEC}(Q, D)$ are conjugate TECs.

Example 6. Letting $P$ be the dots surrounded by circles in Figure 2B, two members $Q_{2}$ and $Q_{6}$ of the conjugate TEC are indicated by dashed lines. Another example: with $P_{1}$ as shown in Figure 3B, a member $Q_{1}$ of the conjugate TEC is indicated by solid bounding lines.

Remark 4. It should be noted that: (1) there might be more than $l$ occurrences of $Q$ in $D$; (2) if $P \subseteq D$ is a maximal translatable pattern, it does not follow that the conjugate pattern $Q$ or one of its translations are maximal translatable patterns. Examples of both of these statements are shown in Figure 3B. (1) If we let $D$ be the set of all points in Figure 3B, and $P_{1}$ be as shown, then the conjugacy array of $P_{1}$ contains two rows and three columns, and the pattern $Q_{1}$ is defined by the first column, contains two points and has at least three occurrences. We see from the unfilled dots in Figure $3 \mathrm{~B}$ that $Q_{1}$ has four occurrences. (2) Now let $D$ be the set of crosses only in Figure $3 \mathrm{~B}$, so that $P_{1}$ is a maximal translatable pattern. The points in the bottom-right corner of Figure $3 \mathrm{~B}$, however, prevent $Q_{1}$ or one of its translations being maximal translatable patterns.

Theorem 2. Let the pattern $P$ have $m$ occurrences in the point set D, contain $l$ points, and be maximal with respect to its translational equivalence class. Then there exists a conjugate pattern $Q$ that has l occurrences in $D$, contains $m$ points, and is maximal with respect to its translational equivalence class.

Proof. Let $\boldsymbol{J}_{P, D}$ denote the conjugacy array for the pattern $P$ in the point set $D$, and let $Q=\left\{\boldsymbol{p}_{1,1}, \boldsymbol{p}_{2,1}, \ldots, \boldsymbol{p}_{m, 1}\right\}$, as in Definition $\%$. 
We suppose that $Q$ is not maximal with respect to its translational equivalence class (MTEC) and try to derive a contradiction. If $Q$ is not MTEC, then by definition there exists $\boldsymbol{e}_{1} \in D \backslash Q$ such that $Q \cup\left\{\boldsymbol{e}_{1}\right\}$ has as many occurrences in $D$ as $Q$. We can show occurrences of $Q \cup\left\{\boldsymbol{e}_{1}\right\}$ as columns in the array

$$
\boldsymbol{J}_{P, D}^{\prime}=\left(\begin{array}{cccc}
\boldsymbol{p}_{1,1} & \boldsymbol{p}_{1,2} & \cdots & \boldsymbol{p}_{1, l} \\
\boldsymbol{p}_{2,1} & \boldsymbol{p}_{2,2} & \cdots & \boldsymbol{p}_{2, l} \\
\vdots & \vdots & \ddots & \vdots \\
\boldsymbol{p}_{m, 1} & \boldsymbol{p}_{m, 2} & \cdots & \boldsymbol{p}_{m, l} \\
\boldsymbol{e}_{1} & \boldsymbol{e}_{2} & \cdots & \boldsymbol{e}_{l}
\end{array}\right) .
$$

But now $E=\left\{\boldsymbol{e}_{1}, \boldsymbol{e}_{2}, \ldots, \boldsymbol{e}_{l}\right\}$ is an extra occurrence of $P=\left\{\boldsymbol{p}_{1,1}, \boldsymbol{p}_{1,2}, \ldots, \boldsymbol{p}_{1, l}\right\}$, and so $P$ has $m+1$ occurrences in $D$. This is a contradiction to $P$ having $m$ occurrences in $D$, from which it follows that $Q$ must be MTEC.

By a similar argument (constructing a new column instead of a new row), it follows that $Q$ has exactly $l$ occurrences.

Remark 5. Theorem 2 establishes an important relationship between MTECs and conjugate patterns, showing that the conjugate patterns of MTEC patterns are themselves MTEC.

\section{Summary and possible directions for future work}

Over the past decade or so, there has been a growing interest in using point sets to represent music, especially in computational musicology and music information retrieval. One of the advantages of this approach is that it is well-suited to representing "unvoiced" polyphonic music (e.g., keyboard music). Another benefit is that it allows for the efficient, automatic discovery of maximal translatable patterns (MTPs) that often correspond closely to perceptually salient repeated musical patterns, such as motives, themes and sections. However, MTPs also quite commonly suffer from the problem of isolated membership.

The contribution of this paper is theoretical primarily, providing a deeper understanding of the mathematical properties of MTPs and a new class of patterns that are maximal with respect to their translational equivalence classes (MTEC). We have shown that a pattern is MTEC if and only if it is the intersection of the MTPs of its translators (Theorem 1). We have also shown that MTEC patterns have MTEC conjugates (Theorem 2).

At present it is unclear whether the calculation of MTECs represents a viable alternative solution to the problem of isolated membership. While the example in Figure 2 is encouraging, generating all the MTEC patterns for even a modestlysized piece of music would be impractical, since the number of MTEC patterns is $O\left(2^{k}\right)$ where $k$ is the number of MTPs, and even a piece of around 500 notes may well have tens of thousands of MTPs. Designing a practical algorithm for generating only the perceptually salient MTEC patterns is therefore an interesting problem for future research. Once such an algorithm has been developed, it 
will be possible to explore more rigorously whether perceptually salient musical patterns correspond more closely to MTEC patterns or MTPs.

Acknowledgments. This research was undertaken during the first author's EPSRC-funded studentship at The Open University, UK. He would like to thank Robin Laney, Alistair Willis, and Paul H. Garthwaite for their time and expert advice. The first author's work is currently supported by the Austrian Science Fund (FWF) under project number Z159 (Wittgenstein Grant). We would also like to thank the two anonymous reviewers for their comments.

\section{References}

1. Meredith, D., Lemström, K., Wiggins, G.A.: Algorithms for discovering repeated patterns in multidimensional representations of polyphonic music. Journal of New Music Research 31 (2002) 321-345

2. Collins, T., Thurlow, J., Laney, R., Willis, A., Garthwaite, P.H.: A comparative evaluation of algorithms for discovering translational patterns in Baroque keyboard works. In: 11th International Symposium on Music Information Retrieval, Utrecht (2010) 3-8

3. Clifford, R., Christodoulakis, M., Crawford, T., Meredith, D., Wiggins, G.A.: A fast, randomised, maximal subset matching algorithm for document-level music retrieval. In: 7th International Symposium on Music Information Retrieval, Victoria, Canada (2006) 150-155

4. Collins, T.: Improved methods for pattern discovery in music, with applications in automated stylistic composition. PhD thesis, The Open University (2011)

5. Collins, T., Laney, R., Willis, A., Garthwaite, P.H.: Modeling pattern importance in Chopin's mazurkas. Music Perception 28 (2011) 387-414

6. Lemström, K.: Towards more robust geometric content-based music retrieval. In: 11th International Symposium on Music Information Retrieval, Utrecht (2010) $577-$ 582

7. Lubiw, A., Tanur, L.: Pattern matching in polyphonic music as a weighted geometric translation problem. In: International Symposium on Music Information Retrieval, Barcelona (2004) 154-161

8. Meredith, D.: The ps13 pitch spelling algorithm. Journal of New Music Research 35 (2006) 121-159

9. Meredith, D.: Point-set algorithms for pattern discovery and pattern matching in music. In: Proceedings of the Dagstuhl Seminar on Content-Based Retrieval (No. 06171), Internationales Begegnungs- und Forschungszentrum für Informatik (IBFI), Schloss Dagstuhl, Germany (2006)

10. Meredith, D.: Computing pitch names in tonal music: a comparative analysis of pitch spelling algorithms. D.Phil. thesis, University of Oxford (2007)

11. Romming, C.A., Selfridge-Field, E.: Algorithms for polyphonic music retrieval: the Hausdorff metric and geometric hashing. In: 8th International Symposium on Music Information Retrieval, Vienna (2007) 457-462 